\title{
Use of High Repetition Rate and High Power Lasers in Microfabrication: How to Keep the Efficiency High?
}

\author{
Gediminas RAČIUKAITIS, Marijus BRIKAS, Paulius GEČYS, Bogdan VOISIAT, Mindaugas GEDVILAS \\ Laboratory for Applied Research, Institute of Physics, Savanoriu Ave. 231, LT-02300 Vilnius, \\ Lithuania \\ E-mail: graciukaitis@ar.fi.lt
}

\begin{abstract}
Effects related to the use of high power and repetition rate lasers in ablation of metals and silicon were investigated. Numeric simulation and experiments were performed to find out conditions of the efficient use of laser energy. Accumulation of defects lowers the ablation threshold when a high pulse-repetition rate is applied. An optimum beam waist exists for certain pulse energy to maximize the ablation but the waist does not allow achieving a high processing accuracy. Energy efficiency of the laser processing falls down when pulse energy exceeds the material dependant limit. Plasma shielding was assumed to be the main limiting factor in processing efficiency of metals with the high power picosecond lasers. Intelligent control of process parameters is required to keep optimal conditions for the material removal by laser ablation.
\end{abstract}

DOI: $10.2961 /$ jlmn.2009.03.0008

Keywords: ablation threshold, accumulation effects, ablation rate, picosecond laser, metals

\section{Introduction}

Requirements of modern micro- and nanotechnologies for the material processing can be fulfilled by laser fabrication. It offers versatile methods for cutting, drilling, ablation, internal modification of a variety of engineering materials. Laser microfabrication has particular benefits compared to other fabrication technologies (chemical wet etching, electro-erosion, etc.) because it is a non-contact process with high accuracy, repeatability and flexibility. Most of engineering materials can be processed with lasers, especially of ultra-short pulse duration. Meanwhile the achieved processing quality meets industrial demands; however, processing speed is still far from an economical industrial use [1]. As the acceptance of the laser microfabrication technology to practical implementation in an industrial process is often measured by the amount of material evaporated during the time span in $\mathrm{mm}^{3} / \mathrm{min}$, investigations of the ablation process efficiency with the real high-power and high-repetitionrate lasers remain relevant at present.

Using ultra-short pulse lasers, the ablation process can be easily controlled because the material is removed in small quantities. A lot of laser pulses are required to remove a considerable amount of the material. The typical method of machining with ultra-short laser pulses is acieved by raster scanning, or the machining of sequentially overlapping linear trenches [2]. The main obstacle to a wide spread of laser micro-technologies, despite their unique possibilities, is the fabrication efficiency. The way of increasing the efficiency of laser microfabrication is to increase the pulse-repetition rate and the average power of the laser.

It is known from the experiments with dielectrics that the surface irradiated with multiple pulses of a high-power laser could be damaged at pulse energies far below the single-shot ablation threshold [3]. This is an important aspect to be considered when using optical components in highpower laser systems. The ablation threshold is also an important parameter in the laser processing because it defines minimal requirements for energetic parameters of the laser. The ablation threshold $F_{t h}$ of the materials was reduced after irradiation of the surface with a sequence of laser pulses from a low-repetition-rate laser. Although the absorbed laser energy is too low to evaporate the material (below the threshold), it introduces structural defects. Accumulation of defects is related to chemical and structural changes in the material [3], [4]. Defects, which require the incubation period, facilitate evaporation of the material by next coming laser pulses. The defects impact on the main parameter of laser processing - the ablation threshold. Using high-repetition-rate lasers the effects should be more evident.

The ratio of the actual laser fluence to the ablation threshold is a measure of the material excitation. As the fluence of the pulse with fixed energy depends on the beam spot, it can be easily controlled by variation in focusing. A simple model of [2] has shown that geometrical optimization of the ablation efficiency is possible. The model is valid for fluences close to the ablation threshold as the energy coupling might be affected by products of the ablation itself. When excitation of the material is high, the ablated mass becomes thermally ionized and opaque to the incident laser radiation [5]. The laser plasma formation has been an objective of numerous investigations during the long time from the laser invention [5-10]. A typical time required for ignition of plasma with an ultra-short laser pulse is $5-10 \mathrm{ps}$ [8]. Therefore, plasma might have an effect on energy coupling to the target even when picosecond lasers are applied. The ablation with ultra-short laser pulses without the plasma generation took place when the laser fluence was below $5 F_{t h}$ [9], while a typical laser fluence for plasma ignition with nanosecond pulses is in the range of 5- 
$10 \mathrm{~J} / \mathrm{cm}^{2}$ for metals [5] and silicon [11]. The upper limit for metal processing with ultra-short laser pulses is expected to be at a few hundred $\mathrm{kHz}$ due to plasma shielding [1].

Affordable machining efficiency can be maintained with the lasers of an appropriate repetition rate and the mean power of the laser should exceed the minimal value of a few watts. Here we discuss how properties of the material, beam and ambience might have influence on the efficient use of laser energy.

\section{Experimental}

The picosecond laser PL10100 (Ekspla) generating 10 ps-long pulses at repetition rates from 50 to $100 \mathrm{kHz}$ was used in experiments. The output power was up to $10 \mathrm{~W}$.

The experiments on defect accumulation effects were performed at the pulse repetition rate of $1 \mathrm{kHz}$ in order to eliminate the effects related to plasma shielding and thermal phenomena. The required pulses at a reduced rate were selected from the outgoing pulse train of the $100 \mathrm{kHz}$ repetition rate with the Pockel's cell acting as a fast shutter. The same Pockel's cell was used to control the pulse energy of the picosecond laser. The laser beam was expanded to the diameter of $4 \mathrm{~mm}\left(1 / \mathrm{e}^{2}\right)$ and directed to the galvoscanner ScanGine 14 (ScanLab). Laser radiation was focused on the sample by telecentric lens with the focal length of $160 \mathrm{~mm}$. The spot size was $20 \mu \mathrm{m}$. The scanner was controlled using SAM Light software (Scaps).

The experiments were performed on the silicon wafer and thin sheets of metals: aluminum $(20 \mu \mathrm{m}$ and $40 \mu \mathrm{m}$ thick), copper ( $34 \mu \mathrm{m})$ and stainless steel $304(400 \mu \mathrm{m}$ and $300 \mu \mathrm{m})$. Craters were formed in the surface when laser fluence was above the ablation threshold. Separate craters were ablated with 110,100 and 1000 laser pulses at a given laser pulse energy, and experiments were repeated using a set of laser pulse energies.

For evaluation of the ablation threshold we used the method introduced by J.M. Liu [12] valid when the Gaussian beams are applied. The diameters of craters were measured through the optical microscope and plotted versus laser pulse energy used to ablate the crater. The threshold was estimated from the relationship between the laser fluence $F_{0}$ and the diameter $D$ of a crater etched with a pulse:

$$
D^{2}=2 \omega_{0}^{2} \ln \left(\frac{F_{0}}{F_{\text {th }}}\right)
$$

where $\omega_{0}$ denotes the beam waist and $F_{t h}$ is the threshold fluence. Linear fitting of the data was performed in representation of experimental data as $D^{2}=f\left(\ln \left(E_{p}\right)\right)$. The waist radius (a laser spot) was estimated at the first step from a slope of the fitting line, and value of the laser pulse energy $E_{p}$ was converted to the laser fluence.

The ablation rate was estimated in bulk metal specimens. Rectangular cavities with the lateral dimension of $1 \times 1 \mathrm{~mm}^{2}$ were milled in the metals with multiple laser pulses. The laser power was varied between the experiments, while the scanning speed, hatch and the number of scans were kept constant. 8 million pulses with the pulse energy in the range of $10-50 \mu \mathrm{J}\left(3-16 \mathrm{~J} / \mathrm{cm}^{2}\right)$ were applied. The depth of laser-milled housings was measured using an optical microscope, and the ablated volume was calculated.

\section{Results}

\subsection{Accumulation effects \& ablation threshold}

The ablation threshold for stainless steel, copper, silicon and aluminum irradiated with the picosecond laser was estimated from the crater diameters using the J.M Liu method [12] and results are given in Table 1, together with the data of Pantsar et al. [14] and Gamali et al. [15] presented in separate columns for comparison.

Table 1 Ablation threshold of metals for the 10 ps pulse duration.

\begin{tabular}{|l|llll|l|l|}
\hline Material & \multicolumn{7}{|c|}{ Ablation threshold, $\mathrm{J} / \mathrm{cm}^{2}$} & {$[14]$} & {$[15]$} \\
\hline & 1 & 10 & 100 & 1000 & & \\
& pulse & pulses & pulses & pulses & & \\
\hline SS304 & 0.5 & 0.2 & 0.1 & 0.04 & 0.15 & 0.19 \\
Al & 0.85 & 0.47 & 0.16 & 0.15 & 0.3 & 0.17 \\
Cu & 1.73 & 0.74 & 0.50 & 0.33 & 0.35 & 0.23 \\
\hline
\end{tabular}

We used the incident laser power (pulse energy) instead of the absorbed one in evaluations as was also done in [14, 15]. It is not correct regarding parameters of the material because most of the energy was reflected by the metal surface. Metals reflect about $70-99 \%$ of laser radiation in the near infrared range. It is technically difficult to measure the laser energy coupled to the workpiece but in our case the ablation threshold was sensitive to surface finishing conditions. No special attempts were made to prepare the surface of specimens before experiments, such as chemical or electro-chemical polishing [13].

According to the accumulation model of Jee et al. [13], the ablation threshold and the number of laser pulses used to ablate a crater are related by an equation:

$$
F_{t h}(N)=F_{t h}(1) N^{S-1},
$$

where $0<S \leq 1$ is the accumulation coefficient, which describes incubation of defects after laser irradiation. $S=1$ means that no incubation appears and the ablation threshold does not depend on the number of laser pulses. A typical value for metals is $S=0.8-0.9$. If the parameter $S$ is larger than 1 , the specimen surface is hardened by laser irradiation. The ablation threshold can be influenced by the initial state of the surface. The surface roughness and contamination increase absorption, and therefore the energy input to the material. The ablation thresholds from Table 1 were plotted in Fig. 1 as $\log \left(F_{t h}(N) * N\right) \sim f(\log (N))$. Linear approximation was performed according to equation (2). Slope of the lines is determined by the accumulation parameter $S$.

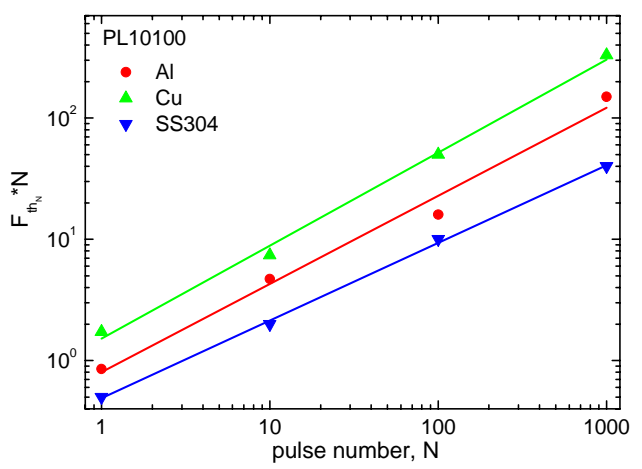

Fig. 1 Ablation threshold versus laser pulse number. 


\subsection{Laser beam focusing \& ablation efficiency}

Modeling and experiments were performed to establish relations between the laser ablation efficiency in terms of the material removal rate with parameters of the laser beam: the spot size, pulse energy or fluence. The modeling was based on the work of Furmanski et al. [2]. The model is a simplified view to the laser ablation. It does not take into account any reflection from the surface, including tilted crater walls. The whole laser energy is coupled in a narrow layer of the material according to the Beer law. Any energy losses due to heat conduction and plasma absorption are excluded. The assumption is valid quite well in case of ultra-short pulses when the heat diffusion during pulse duration is less than the absorption depth. The analysis was performed in order to determine relations between the laser and material parameters as well as the efficiency and precision of laser fabrication by ablation.

The depth of the crater $z$, ablated with a single laser pulse, can be found as [2]:

$$
z(x, y)=\frac{1}{\alpha_{e f f}} \cdot \ln \frac{F(x, y)}{F_{t h}}=\delta \cdot \ln \frac{F(x, y)}{F_{t h}},
$$

where $\delta$ is the effective absorption depth; $F_{t h}$ is the ablation threshold, $F(x, y)$ is the laser fluence spatial distribution in the XY plane.

The diameter of the crater near the surface is fluencedependent as in equation (1) if the Gaussian beam is applied. Laser fluence in the center of the beam distribution is related to the pulse energy and the beam waist as:

$$
F_{0}=\frac{2 E_{\mathrm{p}}}{\pi w_{0}^{2}},
$$

where $E_{\mathrm{p}}$ is the laser pulse energy. The volume of the crater can be calculated by integrating the crater profile (3):

$$
V=\frac{\pi w_{0}^{2} \delta}{4}\left(\ln \frac{F_{0}}{F_{\text {th }}}\right)^{2} \text {. }
$$

The volume of ablated material is maximal when the laser beam is focused to an optimal spot with the waist, which depends on the pulse energy and the ablation threshold:

$$
w_{0 \max }^{2}=\frac{2 E_{\mathrm{p}}}{e^{2} \pi F_{\mathrm{th}}} .
$$

Laser microfabrication is based on the use of multiple laser pulses to ablate the material. When a burst of laser pulses is applied together with scanning, every new pulse hits the workpiece surface at a different place. The scanning can be expressed by a shift between centers of laser spots $\Delta x$ or by a beam overlap in $\%$ as follows $\left(2 w_{0^{-}}\right.$ $\Delta x) / 2 w_{0}$.

If a shift between every two laser pulses is $\Delta x$, the laser fluence of the $n$ - th pulse at initial point can be written as:

$$
F(x=0, y)=F_{0} \exp \left(-2\left(\frac{n \Delta x}{w_{0}}\right)^{2}\right)\left(-\frac{2 y^{2}}{w_{0}^{2}}\right) .
$$

The depth of the final crater (trench) increases by applying the $n$-th laser pulse (taking into account its shift) by amount of $z_{n}$ :

$$
z_{n}(y)=\delta\left[\ln \frac{F_{0}}{F_{\mathrm{th}}}-2\left(\frac{n \Delta x}{w_{0}}\right)^{2}-\frac{2 y^{2}}{w_{0}^{2}}\right] .
$$

The profile of the trench made by partially overlapping laser pulses can be calculated by using the formula:

$$
Z(y)=\sum_{-N}^{N} z_{n}(y)=\frac{\Delta x \delta \sqrt{2}}{3 w_{0}} \sqrt{\ln \frac{F_{0}}{F_{\mathrm{th}}}-\frac{2 y^{2}}{w_{0}^{2}}}\left\{\frac{2 w_{0}^{2}}{\Delta x^{2}}\left[\ln \frac{F_{0}}{F_{\mathrm{th}}}-\frac{2 y^{2}}{w_{0}^{2}}\right]-1\right\} .
$$

Summation is taken for the shifted laser pulses with the fluence above the ablation threshold at the initial place. The depth of the resulting trench depends on the pulse energy and the beam focusing when the shift between pulses is kept constant. Cross-section of the trench might be used as a measure of the laser ablation efficiency:

$$
S=\frac{\delta \pi}{6 \Delta x} \ln \frac{F_{0}}{F_{\text {th }}}\left(\frac{3 w_{0}^{2}}{2} \ln \frac{F_{0}}{F_{\text {th }}}-\Delta x^{2}\right) .
$$

The evaporation rate (volume per time) can be estimated when the pulse repetition rate $R_{\text {rep }}$ and the shift between pulses are taken into account:

$$
\frac{\mathrm{d} V}{\mathrm{~d} t}=R_{\text {rep }} S \Delta x=R_{\text {rep }} \frac{\delta \pi}{6} \ln \frac{F_{0}}{F_{\text {th }}}\left(\frac{3 w_{0}^{2}}{2} \ln \frac{F_{0}}{F_{\text {th }}}-\Delta x^{2}\right) .
$$

The relationship between the evaporation rate and the laser pulse energy as well as the fluence is non-linear. By varying focusing of the beam is it possible to reach a maximum in the evaporation rate of the material (Fig.2).

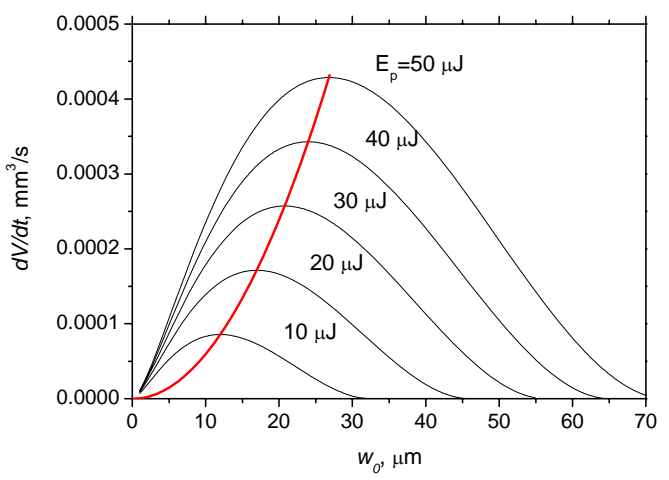

Fig. 2 Material removal rate at ablation of the trench by a burst of laser pulses depending on the laser beam waist at fixed pulse energies. $F_{\text {th }}=0.6 \mathrm{~J} / \mathrm{cm}^{2}, \delta=0.038 \mu \mathrm{m}$, $\mathrm{R}_{\mathrm{rep}}=50 \mathrm{kHz}, \mathrm{dx}=0.1 \mu \mathrm{m}$.

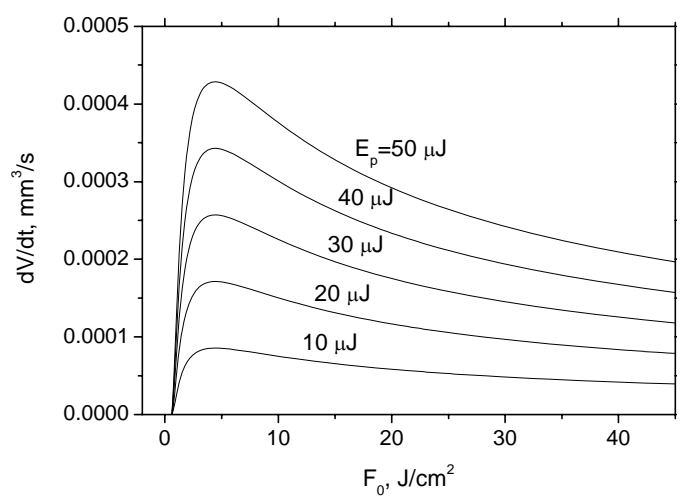

Fig. 3 Material removal rate at ablation of the trench by burst of laser pulses as a function of laser fluence in the beam center at fixed pulse energies. The fluence is varied by focusing. $\mathrm{F}_{\mathrm{th}}=0.6 \mathrm{~J} / \mathrm{cm}^{2}, \delta=0.038 \mu \mathrm{m}, \mathrm{R}_{\mathrm{rep}}=50 \mathrm{kHz}$, $\mathrm{dx}=0.1 \mu \mathrm{m}$.

For every given set of laser pulse energy $E_{\mathrm{p}}$, the ablation threshold $F_{\text {th }}$ and the distance between subsequence laser pulses $\Delta x$, it is possible to define the beam waist when the evaporation rate is maximal (Fig.3). If the shift between subsequent laser pulses is much less than the beam radius 
$\left(\Delta x^{2}<<w_{0}^{2}\right)$, the optimal beam waist is the same as in case of the single pulse ablation (6). The most efficient material removal takes place when the laser fluence is equal to:

$$
F_{0 \text { max }}=e^{2} F_{\text {th }} \approx 7.4 F_{\text {th }} \text {. }
$$

The expression for the maximal evaporation rate at the optimal laser beam focusing can be written by substitution of equation (6) into equation (11):

$$
\left(\frac{\mathrm{d} V}{\mathrm{~d} t}\right)_{\max }=R_{\mathrm{rep}} \frac{2 \delta E_{\mathrm{p}}}{e^{2} F_{\mathrm{th}}}\left(1-\frac{\Delta x^{2}}{w_{0 \text { max }}^{2}}\right) \approx R_{\mathrm{rep}} \frac{2 \delta E_{\mathrm{p}}}{e^{2} F_{\mathrm{th}}} .
$$

The evaporation rate depends on material properties (absorption depth and ablation threshold) and parameters of the laser beam (pulse energy, repetition rate). The pulse energy and the repetition rate linearly affect the evaporation rate. Therefore, these laser parameters are topmost important for scaling the efficiency of laser microfabrication.

Experimental verification of the modeling results was performed by ablating trenches in stainless steel at variable focusing. The depth profiles of the trenches were measured with a stylus profiler. At a given scanning speed, the evaporation rate $d V / \mathrm{d} t$ was calculated and it is shown as a function of the beam waist at the constant pulse energy (Fig. 4) and as a function of the laser fluence which was controlled by defocusing (Fig. 5).

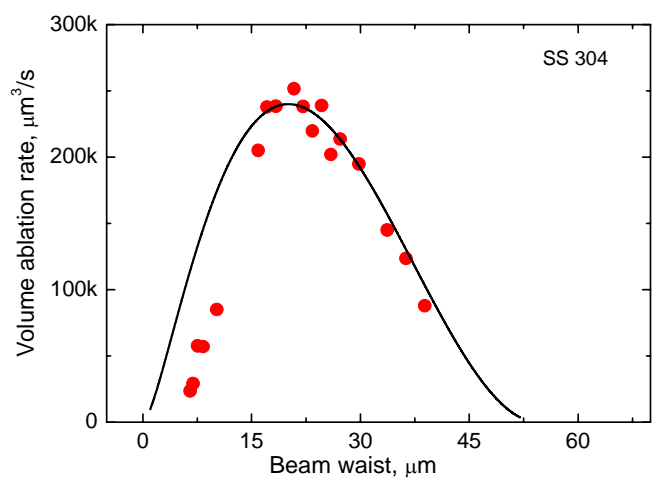

Fig. 4 Volume removal rate in $\mathrm{mm}^{3} / \mathrm{s}$ of stainless steel as a function of beam waist. Pulse energy was $28 \mu \mathrm{J}$ at $50 \mathrm{kHz}$. $\mathrm{F}_{\text {th }}=0.6 \mathrm{~J} / \mathrm{cm}^{2} ; \delta=0.038 \mu \mathrm{m} ; \Delta \mathrm{x}=0.1 \mu \mathrm{m}$.

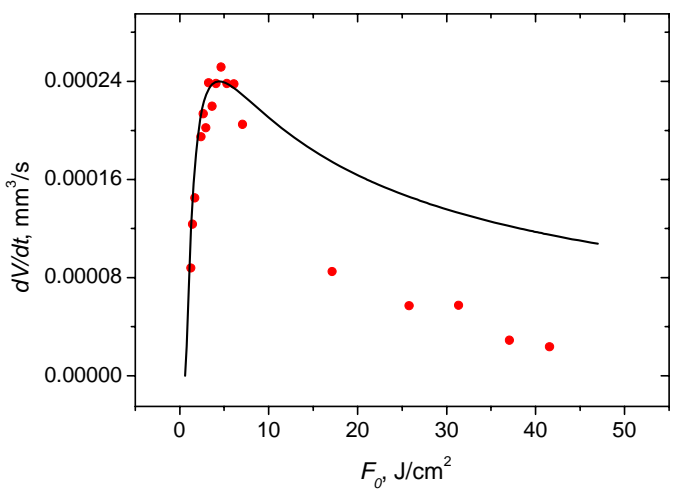

Fig. 5 Volume removal rate in $\mathrm{mm}^{3} / \mathrm{s}$ of stainless steel as a function of laser fluence. The fluence was varied by focusing the beam. Pulse energy was $28 \mu \mathrm{J}$ at $50 \mathrm{kHz} . \mathrm{F}_{\text {th }}=$ $0.6 \mathrm{~J} / \mathrm{cm}^{2} ; \delta=0.038 \mu \mathrm{m} ; \Delta \mathrm{x}=0.1 \mu \mathrm{m}$.

Good correlation between calculated and experimental data was found when the beam waist was large enough or the laser fluence was not too high. Deviations of the experimental data from estimations occurred in an opposite case and could be related to the limitation of the stylus profiler to measure deep and narrow trenches which were formed at the tight focusing and the high laser fluence. The geometrical limitation of the stylus led to the underestimated value of the trench cross-section.

\subsection{Ablation rate \& plasma shielding}

The ablation rate was estimated in bulk metal specimens using picosecond lasers. Rectangular cavities with the lateral dimension of $1 \times 1 \mathrm{~mm}^{2}$ were milled in the metals with multiple ( 8 millions) laser pulses. The depth of the holes was measured using an optical microscope, and an ablated volume was calculated. The depth of laser milled cavities varied from 35 up to $300 \mu \mathrm{m}$. The mean ablation rate in $\mu \mathrm{m} /$ pulse was estimated dividing the cavity volume by the pulse number and the laser spot area. As the milled area was large compared to a laser spot, the shielding effects of a confined crater [18] had no impact on the results. The ablation rate increased with the laser pulse energy but the representation of results did not give information about efficient use of laser energy. Therefore, the data were transformed to the total ablation rate as a depth ablated by a pulse multiplied by the pulse repetition rate. The results versus the mean laser power are presented in Fig.6 and show remarkable deviation from linearity, which means reduction in efficiency of laser ablation at high mean power of the picosecond laser.

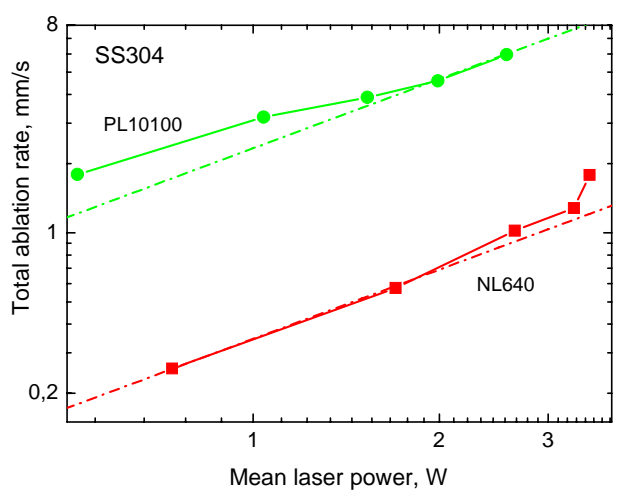

Fig. 6 Ablation rate of the stainless steel 304 as a function of laser pulse energy. PL10100 laser. Dot lines represent linear approximation. Results of ablation with the nanosecond Q-switched laser (NL640) are presented for comparison.

The repetition rate of the picosecond laser was kept constant $(50 \mathrm{kHz})$, while the pulse energy and average power were controlled simultaneously by an attenuator. Saturation in the rise of the total ablation rate was evident at the higher laser power. The ablation efficiency was limited by phenomena that were excited at the high intensity of laser radiation. The results were further transformed into the energetic ablation efficiency in $\mu \mathrm{m}^{3} / \mathrm{mJ}$, the volume of the material ablated with a portion of the laser pulse energy. Both parameters are mean values of action of more than a million laser pulses and represent the real efficiency of laser processing.

Fig. 7 shows experimental data of the energetic ablation efficiency of steel, nickel and aluminum with the picosec- 
ond laser. The data were evaluated at two different pulse repetition rates and are plotted versus the pulse energy. A significant fall in the energetic efficiency occurred using the picosecond laser at a higher power. The evaporation efficiency in $\mu \mathrm{m}^{3} / \mathrm{mJ}$ was higher for the pulse repetition rate of $50 \mathrm{kHz}$ (filled dots) compared to $100 \mathrm{kHz}$ (open dots) at the same pulse energy in case of all examined metals, except aluminum. The repetition rate had no effect on the energetic ablation efficiency in aluminum. Nickel showed a large drop in the efficiency in a narrow range of pulse energies.

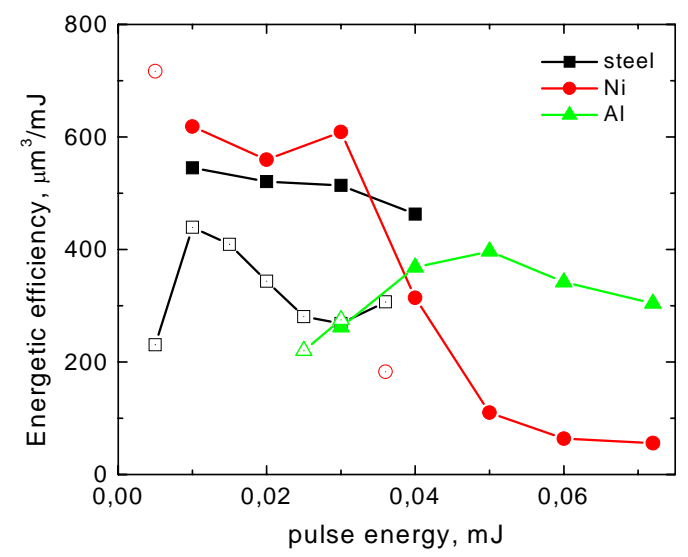

Fig. 7 Ablation efficiency in metals with the picosecond laser at the $50 \mathrm{kHz}$ (filled dots) and $100 \mathrm{kHz}$ (open dots) repetition rate.

\section{Discussions}

The ablation threshold is the most important parameter of the material in laser ablation. It was found to be dependent on the number of pulses irradiating the same place of the specimen. Every laser pulse, even with the energy below the evaporation threshold, induced structural alterations. The deformations of the metal surface occurred under irradiation with the laser pulse. Defects on the surface were accumulated at the fluences below the single-pulse threshold. Therefore, the lower laser pulse energy was required for initiation of the material removal in the multishot regime. As a result, the ablation threshold decreased with the increase in the number of pulses.

A significant change in the ablation threshold was observed for all investigated metals depending on the number of laser pulses applied to evaporate the crater. We did not use any preparation of the specimens in experiments, while an electro-polishing was applied in [15]. Accumulation effects depended on the initial condition of the surface. Apparently, the surface of the rolled metal sheet in our case was in a meta-stable state and irradiation with a sequence of laser pulses triggered the defect formation. Lowering of the ablation threshold might be useful in laser material processing using high-repetition-rate lasers which usually generate the low energy pulses.

The ablation threshold of metals was investigated by many groups but there was no systematic how irradiation with multiple laser pulses influences that important parameter of material. Gamali et al. in [15] estimated the ablation threshold of metals in vacuum and in the air using a laser with the pulse duration of $12 \mathrm{ps}$ and the high pulse repetition rate of $4.2 \mathrm{MHz}$. The values of $0.17 \mathrm{~J} / \mathrm{cm}^{2}$ for aluminum, $0.23 \mathrm{~J} / \mathrm{cm}^{2}$ for copper and $0.19 \mathrm{~J} / \mathrm{cm}^{2}$ for steel are close to our values when the large number of pulses $(\mathrm{N}=1000)$ was applied. The single pulse ablation threshold of copper and stainless steel was found to be $0.58 \mathrm{~J} / \mathrm{cm}^{2}$ and $0.21 \mathrm{~J} / \mathrm{cm}^{2}$, respectively, when the laser pulse duration was $150 \mathrm{fs}$ [16]. The thresholds fell down to $0.55 \mathrm{~J} / \mathrm{cm}^{2}$ and $0.13 \mathrm{~J} / \mathrm{cm}^{2}$, respectively, when 100 pulses were applied. The values and accumulation coefficient are close to those estimated in this work for 10 ps pulse duration.

For the same pulse energy, the fluence can be varied by changing focusing of the beam. Therefore, simple geometrical modeling the effect of focusing on the material removal efficiency was carried out. Any effects that could have an influence on the energy coupling to the material or its removal were not taken into account. The situation was realized when low fluences just above the ablation threshold were applied and the aspect ratio of the trench was small.

The optimal focusing conditions for stainless steel were estimated. The best results for laser pulses with the energy of $28 \mu \mathrm{J}$ were achieved when the beam was close to $20 \mu \mathrm{m}$ in radius $\left(1 / \mathrm{e}^{2}\right)$. The optimal beam radius increases with the pulse energy. The efficient material removal requires moderate laser fluences. When the pulse energy is high, the spot size becomes large. It can be too large in order to achieve the required precision of fabrication in the range of 1-2 $\mu \mathrm{m}$ or below. Two solutions might be applied:

- Low pulse energies can be used to machine the small features. The excessive laser energy can be applied by splitting the beam and using multi-beam parallel processing;

- Fabrication process can be organized with variable focusing and the pulse energy control. The initial "rough" fabrication should be performed at the maximal pulse energy and the optimal beam focusing in order to achieve the most efficient material removal rate. Later, the reduced pulse energy and focusing to a small spot can be used to tune the shape to the required precision.

An increase in the incident laser fluence on the workpiece surface intensified the material removal. High intensity of laser radiation facilitates heating of the material to very high temperatures, when ionized atoms are transformed into plasma [5]. Plasma effectively absorbs laser radiation shielding of the specimen [8]. It begins to form with delay of 5-10 ps after the irradiation start from the evaporated material. Even for the laser pulses as short as $10 \mathrm{ps}$, the plasma can have an impact on the energy transfer from the beam to the material.

The intensity of the absorbed laser radiation used in experiments was estimated and compared to the intensity of plasma ignition of metals $\left(2 * 10^{13} \mathrm{~W} / \mathrm{m}^{2}\right)$ [19],[20]. In case of the picosecond laser, the intensity was much higher than the plasma ignition limit in the whole range of laser power, and the rise in intensity was synchronous with a fall in the ablation efficiency. Therefore, we suppose that the limiting factor in the energetic ablation efficiency with the picosecond laser was plasma formation above the specimen surface.

Every investigated metal showed a specific pulse energy when the energetic ablation efficiency was maximal. The pulse energy in Fig. 7 corresponds to the incident laser energy. Aluminum has reflectivity of $91 \%$ at the $1064 \mathrm{~nm}$ 
wavelength, while that of nickel and steel is about $70 \%$. Therefore, aluminum required a three times higher incident pulse energy to absorb the same amount of laser power compared to nickel. Adjustment of the efficiency of lasers according to the absorbed laser energy led to a close range of laser parameters for the optimal use of the laser power.

In contrast to the most research on laser ablation in metals, the laser milling in this work was performed on a large area compared to the laser spot by scanning the laser beam. Therefore, the ablation products remaining in the air above the specimen cannot absorb the laser beam significantly contrary to the case of percussion drilling [8]. The high repetition rate was found to be a problem when the repetition rate exceeded a few hundreds of $\mathrm{kHz}$ in metal drilling experiments [21]. Our experiments were limited to lower repetition rates and we cannot confirm the results for large-area ablation.

The direct increase in the pulse energy can be an inefficient way to reach the higher ablation rate. Besides the defect generation with high-repetition-rate lasers, a significant amount of absorbed energy remains in the material. The heat accumulation is another feature of laser processing with high-repetition-rate lasers. Splitting the beam into a few beams might be a solution with high pulse energy lasers.

\section{Conclusions}

Three types of effects which had an influence on the material removal rate and thus on the laser processing efficiency were investigated.

The ablation threshold of metals and silicon depended on the number of pulses affecting the same area. The reduction in the ablation threshold by irradiation with a series of laser pulses can be useful in application of the highrepetition-rate lasers with the low pulse energy in order to increase the processing efficiency. At a constant scanning speed, the beam overlap increases with the repetition rate. As a consequence, the reduced ablation threshold is able:

- to make easier the material removal with the lower energy pulses that are usually generated with high repetition rate lasers;

- to remove more material by one laser pulse of the same fluence because the ablation rate depends on the above-threshold fluence.

The volume ablation rate is a non-linear function of the pulse energy. Numerical simulation of the ablation efficiency was performed using simple relations between the laser and material parameters. An optimum beam waist was estimated for a certain pulse energy to maximize the ablation. However, the beam size was large and did not allow achieving high accuracy. Intelligent control of the beam focusing is required for coarse but efficient as well as fine but slow laser processing.

The energy efficiency of the laser processing fell down when the fluence was above the limit which depended on the material. The density of the absorbed laser energy was close to the plasma formation threshold. The limiting factor in the energy efficiency of ablation with the picosecond laser was plasma formation at the specimen surface. The direct increase in the pulse energy can be an inefficient way to reach a higher ablation rate. Splitting the beam into a few beams might be a solution with high-pulse-energy lasers.

Intelligent control of process parameters is required to keep optimal conditions for the material removal by laser ablation.

\section{Acknowledgments}

The work was supported by the Lithuanian State Science and Studies Foundation under project No V-38/2008. P. Gečys acknowledges the student research fellowship from the Lithuanian Science Council.

\section{References}

[1] J. Konig, S. Nolte, A. Tunnermann, Plasma evolution during metal ablation with ultrashort laser pulses, Optics Express, 13, 10597-10607 (2005).

[2] J. Furmanski, A.M. Rubenchik, M.D. Shirk and B.C. Stuart, J. Appl. Phys., 102, (2007) 073112.

[3] J. Bonse, H. Sturm, D. Schmidt and W. Kautek, Appl. Phys. A71, (2000) 657.

[4] S. Martin, A. Hertwig, M. Lenzner, J. Kruger andW. Kautek, Appl. Phys. A77, (2003) 883.

[5] X. Mao, R.E. Russo, Appl. Phys. A64 (1997 1.

[6] M.A. Shannon, X. Mao, R.E. Russo, Mat. Sci. Engineering, B45 (1997) 172.

[7] J.M. Vadillo, J.M. Fernandez Homero, C.Rodeiguez, J.J. Laserna, Surf. Interface Anal., 27 (1999) 1009.

[8] M. Weikert, Ch. Foehl and F. Dausinger, Proc. 3-rd Int. Symp. on Laser Precision Microfabrication, Proc. SPIE 4830, (2003) 501.

[9] A. Cavalleri, K. Sokolowski-Tinten, J. Bialkowski, M. Schreiner and D. von der Linde, J. Appl. Phys. 85, (1999) 3301

[10] I.I. Beilis, Laser and Particle Beams, 25 (2007) 53.

[11] M.H. Hong, Y.F. Lu, Appl. Phys. A69 (1999) S605.

[12] J.M. Liu, Opt. Lett., 7, (1982) 196.

[13] Y. Jee, M.F. Becker and R.M. Walser, J. Opt. Soc. Am. B, 5, (1988) 648.

[14] H. Pantsar, P. Laakso and R. Penttilä, Proc. 4-th Int. WLT-conference on Laser in Manufacturing, LIM 2007, Munich, June 18-22, 2007, (2007) 613.

[15] E.G. Gamaly, N.R. Madsen, M. Duering, A.V. Rode, V.Z. Kolev and B. Luther-Davies, Phys. Rev. B 71, (2005) 174405.

[16] P.T. Mannion, J. Magee, E. Coyne, G.M. O’Connor and T.J. Glynn, Appl. Surf. Science, 233, (2004) 275.

[17] D. Bauerle, Laser Processing and Chemistry, 3rd edition, Springer (2001).

[18] D. Breitling, A. Ruf and F. Dausinger, Proc. SPIE 5339, (2004) 49.

[19] J.J. Chang, B.E. Warner, E.P. Dragon and M.W. Martinez, Journal of Laser Applications, 10, (1998) 285.

[20] J. Hermann, C. Vivien, A.P. Carricato and C. Boulmer-Leborgne, Appl. Surf. Science, 127-129, (1998) 645.

[21] A. Ancona, F. Roses, K. Rademaker, J.Limpert, S. Nolte, A. Tunnermann, Opt.Express 16 (2008) 8958.

(Received: July 4, 2008, Accepted: November 17, 2009) 